\title{
Small sets with large power sets
}

\section{G.P. Monro}

One problem in set theory without the axiom of choice is to find a reasonable way of estimating the size of a non-well-orderable set; in this paper we present evidence which suggests that this may be very hard. Given an arbitrary fixed aleph $K$ we construct a model of set theory which contains a set $X$ such that if $Y \subseteq X$ then either $Y$ or $X-Y$ is finite, but such that $\kappa$ can be mapped into $S(S(S(X)))$. So in one sense $X$ is large and in another $X$ is one of the smallest possible infinite sets. (Here $S(X)$ is the power set of $X$.)

\section{Preliminaries}

We work in Zermelo-Fraenkel ( $Z F$ ) set theory, without the axiom of choice but with the axiom of foundation.

Notations. If $f: X+Y$ and $A \subseteq X$ then:

$f^{\prime \prime} A=\{y:(\exists x \in A)(f(x)=y)\} ;$

$X * Y$ means that $X$ can be mapped onto $Y$;

$A \Delta B=A \cup B-(A \cap B)$.

We write $|X|$ for the cardinal of $X, S(X)$ for the power set of $X, S_{\kappa}(X)$ for $\{Y \subseteq X:|Y|<\kappa\}, X^{[n]}$ for $\{Y \subseteq X:|Y|=n\} . A_{B}$ is the set of functions from $A$ into $B ; B^{A}=\left|A_{B}\right|$. ' $X$ is finite' means that $X$ has $n$ elements for some $n<\omega$.

Relative constructibility. We write $L$ for Gödel's constructible universe. If $X$ is a transitive set, $L(X)$ is the smallest transitive

Received 24 January 1973. 
proper class which contains $X$ and satisfies $2 F$. It can be shown that, inside $L(X)$, any element of $L(X)$ can be defined from $X$, an element of $L$ and a finite number of elements of $X$.

If $X$ is not transitive, by $L(X)$ we mean $L(\operatorname{TC}(X))$, where

$$
\operatorname{TC}(X)=\{X\} \cup X \cup(U X) \cup(\cup \cup X) \cup \ldots .
$$

$\mathrm{TC}(X)$ (the transitive closure of $X$ ) is the smallest transitive set with $X$ as a member.

Forcing. We follow Shoenfield [3], but adopt a different convention for names in the forcing language: for $x \in M$ we take $x$ as a name for $x$, and we take $G$ as a name for $G$. We adopt from [3] the notation $H_{K}(A, B)$ for

$$
\left\{f: \operatorname{dom}(f) \in S_{K}(A), \operatorname{ran}(f) \subseteq B\right\} .
$$

We note the following symmetry lemma.

LEMMA. Let $M$ be a countable transitive model of ZF, $P \in M$ a notion of forcing, $\pi \in M$ an automorphism of $P$ and $\varphi\left(v_{0}, v_{1}\right)$ a ZF-formula. Then

$$
p \Vdash \varphi(G, x) \leftrightarrow \pi^{-1} p \Vdash \varphi\left(\pi^{\prime \prime} G, x\right)
$$

where $x \in M$ and $p \in P$.

\section{Dedekind-finite sets}

In this section all proofs are carried out in ZF; no use is made of the axiom of choice.

A Dedekind-finite (DF) set is defined to be a set not equinumerous with any of its proper subsets; a DF cardinal is the cardinal of a DF set. In the absence of the axiom of choice infinite DF sets may exist.

LEMMA 2.1. The following are equivalent;

$$
\begin{aligned}
& \text { (i) } X \text { is } \mathrm{DF} \text {; } \\
& \text { (ii) }|X| \neq|X|-1 \text {; }
\end{aligned}
$$

(iii) $\omega \neq|X|$. 
LEMMA 2.2. For an arbitrary set $X, \omega \leq * X$ iff $\omega \leq 2^{X}$.

Proof. This is due to Kuratowski ([4], p. 94-95). We say a set $X$ is quasi-minimal ( $Q M$ ) if $X$ is infinite but has only finite and cofinite subsets ( $Y \subseteq X$ is cofinite if $X-Y$ is finite). Clearly a QM set is $\mathrm{DF}$; in fact it is obvious that $X \mathrm{QM} \rightarrow X$ 利 $\omega$.

The name 'quasi-minimal' (due to Hickman) arises as follows. By Lemma 2.1 (ii) the only cardinal minimal among the infinite cardinals is $w$. However we put an equivalence relation on infinite cardinals thus: $m \equiv m^{\prime}$ if there is $n<w$ such that either $m+n=m^{\prime}$ or $m^{\prime}+n=m$. Write $[m]$ for the equivalence class of $m$, and set $[m] \leq_{1}\left[m^{\prime}\right]$ if $m \leq m^{\prime}$. Then $[m]$ is minimal under the partial order $\leq_{1}$ iff $m=\omega$ or $m$ is QM.

LEMMA 2.3. If $X * \neq \omega$ and $n<\omega$ then $x^{[n]} * \neq \omega$.

Proof. It is straightforward to prove that if $Y * \neq \omega$ and $Z * \neq \omega$ then $y \times z * \neq \omega$. So $x^{n} * \omega$, and trivially $x^{n} * x^{[n]}$.

THEOREM 2.4. Let $X$ be QM, $K$ an aleph.

$$
\begin{aligned}
& \text { (i) } k \leq|x|+k<\omega . \\
& \text { (ii) } k \leq|S(X)|+k<\omega . \\
& \text { (iii) } k \leq|S(S(X))| \rightarrow k \leq 2^{\omega} .
\end{aligned}
$$

Proof. Since $X * \neq$ it follows from Lemma 2.2 that $X$ and $S(X)$ are both $D F$, which establishes $(i)$ and $(i i)$.

We note that $|S(X)|=2 \cdot\left|S_{\omega}(X)\right|$ (this may be seen by associating each infinite subset of $X$ with its complement), and so $|S(S(X))|=\left|S\left(S_{\omega}(X)\right)\right|^{2}$. To establish (iii) it then suffices to show

$$
\kappa \leq\left|S\left(S_{\omega}(X)\right)\right| \rightarrow \kappa \leq 2^{\omega} .
$$

For if $\lambda$ is an aleph, $m$ any infinite cardinal and $\lambda \leq m^{2}$, then $\lambda \leq m$ (see [2], Lemma 6.13, p. 55).

$$
\text { Suppose then that } f: \kappa \rightarrow S\left(S_{\omega}(X)\right) \text { is one-to-one. Set }
$$


$f_{n}(\alpha)=f(\alpha) \cap x^{[n]}$. Now $\left\{f_{n}(\alpha): \alpha \in \kappa\right\} \subseteq S\left(X^{[n]}\right)$, and by Lemma 2.3, $x^{[n]} * \neq$, so by Lemma 2.2, $s\left(x^{[n]}\right)$, and thus $\left\{f_{n}(\alpha): \alpha \in k\right\}$, is DF. However $\left\{f_{n}(\alpha): \alpha \in \kappa\right\}$ has a canonical well-order and so is finite, and can be canonically mapped into $\omega$. Combining these canonical maps for each $n$ yields a one-to-one map of $A=\left\{f_{n}(\alpha): \alpha \in \kappa\right.$ and $\left.n<\omega\right\}$ into $\omega \times \omega$. Now $f(\alpha)$ is determined by $\left(f_{n}(\alpha)\right)_{n<\omega}$, which is an $\omega$-sequence of elements of $A$, and so $f(\alpha)$ can be associated with an element of $\omega(\omega \times \omega)$. It follows that $\kappa$ can be mapped one-to-one into ${ }^{\omega}(\omega \times \omega)$, and so $k \leq 2^{\omega}$.

\section{A large QM set}

In this section we construct the model promised in the abstract. Theorem 2.4 shows why we have to look at $S(S(S(X)))$ rather than some smaller power of $X$.

Let $M$ be a countable transitive model of $Z F+V=L, \kappa$ a (successor aleph) $)^{M}$. Then $M \vDash 2^{\lambda}=\kappa$ for some aleph $\lambda$ of $M$. We take $\left(H_{K}\left(\left(\lambda_{2}\right) \times K, 2\right)\right)^{M}$ as our notion of forcing, with the partial order defined by $p \leq q$ iff $p \supseteq q$. Let $G$ be generic over $M$ with respect to this notion.

LEMMA 3.1. (i) $M$ and $M[G]$ have the same cofinality (cf) function and the same alephs.

(ii) For $\alpha<\kappa$ and $x \in M, \quad\left({ }^{\alpha} x\right)^{M}=\left({ }^{\alpha} x\right)^{M[G]}$.

Proof. We note that $k$ is (regular) ${ }^{M}$. We assume the terms ' $\mu$-closed' and ' $\mu$-chain condition' from [3], $\$ 10$. Our notion of forcing satisfies the $\kappa^{+}$-chain condition (by [3], Lemma 10.3) and is $k$-closed, so our results follow from [3], Lemma 10.2, and Lemma 10.6 and Corollary.

We now work in $M[G]$ unless otherwise stated. For $f \in \lambda_{2}$ set $G(f)=U\{p(f): p \in G\}$. Note that by $p(f)$ we mean 
$\{\langle\alpha, \beta\rangle:\langle\langle f, \alpha\rangle, \beta\rangle \in p\}$. Set $G^{*}=\left\{G(f): f \in \lambda_{2}\right\}$. It can be shown by standard arguments that each $G(f)$ is a member of $\kappa_{2}$ and that if $f \neq g$ then

$$
|\{i<\kappa:(G(f))(i) \neq(G(g))(i)\}|=\kappa .
$$

For $r \epsilon^{K} 2$ set

$$
[r]=\left\{s \in \epsilon^{K}:|\{i<\kappa: s(i) \neq r(i)\}|<k\right\} .
$$

Set $X=\left\{[r]: r \in G^{*}\right\}$. Define for $\alpha<\lambda$,

$Y_{\alpha}=\left\{\left[[G(f)],\left[G\left(f^{\prime}\right)\right]\right\}: f, f^{\prime} \epsilon^{\lambda} 2, f^{\prime}(\alpha)=1-f(\alpha)\right.$ and $f^{\prime}(\beta)=f(\beta)$ for $\left.\beta \neq \alpha\right\}$.

Then $Y_{\alpha}$ is a partition of $X$ into disjoint two-element subsets, and $\alpha \neq \beta \rightarrow Y_{\alpha} \cap Y_{\beta}=\varnothing$. Set

$Y=\{K: X$ is a partition of $X$ into two-element subsets and $K-Y_{\alpha}$ is finite for some $\left.\alpha<\lambda\right\}$, $Z=\left\{\langle K, \alpha\rangle: K \in Y\right.$ and $K-Y_{\alpha}$ is finite $\}$.

The model which is to contain the large $Q M$ set is $N=(L(Z))^{M[G]}$.

The motivation of the construction is as follows. If we set $N^{\prime}=(L(X))^{M[G]}$ it can be readily shown that $N^{\prime} \models X$ is $Q M$. In constructing $N$ we add enough sets to $N^{\prime}$ to make $X$ large in the desired sense, but not enough to destroy the quasi-minimality of $X$.

LEMMA 3.2. (i) $M, N$ and $M[G]$ have the same cf function and alephs.

(ii) For $\alpha<\kappa$ and $x \in M, \quad\left({ }^{\alpha} x\right)^{M}=\left({ }^{\alpha} x\right)^{N}=\left({ }^{\alpha} x\right)^{M[G]}$.

(iii) $N \vdash 2^{\lambda}=\kappa$.

Proof. (i) and (ii) are immediate from Lemma 3.1 , since $M \subset N \subset M[G]$; (iii) is essentially just a special case of (ii).

THEOREM 3.3. $N \models \mathrm{K} \leq|S(S(S(X)))|$. 
Proof. Clearly in $N, Y \subseteq S\left(X^{[2]}\right)$, and also $Z: Y \rightarrow \lambda$ is onto. So $N \neq S(S(X)) * \geq \lambda$. It follows that

$$
N \models 2^{\lambda} \leq|S(S(S(X)))|
$$

and the result follows by Lemma 3.2 (iii).

We now look at $\operatorname{TC}(z)$. If $x \in \operatorname{TC}(z)$ then either $x=2$ or $x=\langle K, \beta\rangle$ for some $K \in Y$ and $\beta<\lambda$ or $x \in Y$ or ... or $x \in M$. It can easily be seen that in all cases either $x=Z$ or $x$ is codable by (at worst) some $Y_{\alpha}$, a finite number of elements of $G^{*}$ and an element of $M$. We recall from $\xi 1$ that inside $N$ every element of $N$ is definable from $\operatorname{TC}(Z)$, an element of $M\left(=(L)^{M[G]}\right)$ and a finite number of elements of $\mathrm{TC}(Z)$. By using the coding just mentioned we have that inside $N$ any element of $N$ is definable from $Z$, a finite number of $Y_{\alpha}^{\prime}$, a finite number of elements of $G^{*}$, and an element of $M$ (as $\mathrm{TC}(Z)$ is definable from $z$ ).

We proceed to a continuity lemma, but first introduce some notation. Suppose $A$ is a set, $s \subseteq A$ and $f: A \rightarrow 2$. We define $f^{S}: A \rightarrow 2$ thus :

$$
f^{s}(a)=f(a) \text { if } a \notin s ; f^{S}(a)=1-f(\alpha) \text { if } a \in s .
$$

LEMMA 3.4. Suppose that

$$
N \vDash \varphi\left(z, y_{\alpha_{1}}, \ldots, Y_{\alpha_{n}}, G\left(f_{1}\right), \ldots, G\left(f_{m}\right), x,[G(f)]\right),
$$

where $x \in M$ and $f \neq f_{i}^{s}$ for $1 \leq i \leq m$ and any $s \subseteq\left\{\alpha_{1}, \ldots, \alpha_{n}\right\}$. Let $g \in \lambda_{2}$, be any function such that $g \neq f_{i}^{s}$ for $1 \leq i \leq m$ and $s \subseteq\left\{\alpha_{1}, \ldots, \alpha_{n}\right\}$. Then

$$
N \vDash \varphi\left(z, Y_{\alpha_{1}}, \ldots, y_{\alpha_{n}}, G\left(f_{1}\right), \ldots, G\left(f_{m}\right), x,[G(g)]\right) \text {. }
$$

Proof. Let $\psi$ be a formula such that

$$
M[G] \vDash \psi(G, y) \leftrightarrow N \vDash \varphi\left(2, y_{\alpha_{1}}, \ldots, Y_{\alpha_{n}}, G\left(f_{1}\right), \ldots, G\left(f_{m}\right), x,[G(f)]\right) .
$$


$\psi$ 'describes' the construction of $Z, Y_{\alpha_{1}}$, and so on, from $G$ and also relativizes $\varphi$ to the class $N$. Here $y \in M$. Take $p \in G$ such that $p \Vdash \psi(G, y)$. Set, for $s \subseteq\left\{\alpha_{1}, \ldots, \alpha_{n}\right\}$,

$$
\begin{aligned}
& A_{s}=\left\{i \in \operatorname{dom}\left(p\left(f^{s}\right)\right): p\left(f^{s}, i\right) \neq\left(G\left(g^{s}\right)\right)(i)\right\}, \\
& B_{s}=\left\{i \in \operatorname{dom}\left(p\left(g^{s}\right)\right): p\left(g^{s}, i\right) \neq\left(G\left(f^{s}\right)\right)(i)\right\} .
\end{aligned}
$$

Then $A_{s}, B_{s} \in S_{K}(K)$ and so by Lemma 3.2 (ii), $A_{s}, B_{s} \in M$.

For $A \in S_{K}(K)$ we define an automorphism $\sigma_{A}$ of $H_{K}(K, 2)$ thus:

$$
\left.\begin{array}{l}
\left(\sigma_{A}(t)\right)(i)=1-t(i) \text { if } i \epsilon \operatorname{dom}(t) \cap A \\
\left(\sigma_{A}(t)\right)(i)=t(i) \text { if } i \in \operatorname{dom}(t)-A
\end{array}\right\} \text { for } t \in H_{K}(K, 2)
$$

and $\operatorname{dom}\left(\sigma_{A}(t)\right)=\operatorname{dom}(t)$.

We define an automorphism $\pi$ of $H_{K}\left(\left(\lambda_{2}\right) \times_{K}, 2\right)$ thus:

$$
\begin{aligned}
& \left.\begin{array}{l}
(\pi p)\left(f^{s}\right)=\sigma_{A_{s}}\left(p\left(g^{s}\right)\right) \\
(\pi p)\left(g^{s}\right)=\sigma_{B_{s}}\left(p\left(f^{s}\right)\right)
\end{array}\right\} \text { for } s \subseteq\left\{\alpha_{1}, \ldots, \alpha_{n}\right\}, \\
& (\pi p)(h)=p(h) \text { for } h \neq f^{s}, g^{s} \text { for any } s \subseteq\left\{\alpha_{1}, \ldots, \alpha_{n}\right\} .
\end{aligned}
$$

Then $\pi \in M$, and

$$
\begin{gathered}
\left(\pi^{-1} p\right)\left(f^{s}\right)=\sigma_{B}\left(p\left(g^{s}\right)\right) \subset G\left(f^{s}\right), \\
\left(\pi^{-1} p\right)\left(g^{s}\right)=\sigma_{A_{s}}\left(p\left(f^{s}\right)\right) \subset G\left(g^{s}\right), \\
\left.\left(\pi^{-1} p\right)(h)=p(h) \subset G(h) \text { (for } h \neq f^{s}, g^{8}\right) .
\end{gathered}
$$

It follows that $\pi^{-1} p \in G$. Now $p \| \psi(G, y)$, so by the symmetry lemma of $51, \pi^{-1} p \Vdash \psi(\pi " G, y)$, whence 


$$
M[G] \vDash \psi(\pi " G, y) .
$$

Now

$$
\begin{gathered}
\left(\pi^{\prime \prime} G\right)\left(f^{s}\right)=\sigma_{A_{s}}\left(G\left(g^{s}\right)\right), \\
\left(\pi^{\prime \prime} G\right)\left(g^{s}\right)=\sigma_{B_{s}}\left(G\left(f^{s}\right)\right), \\
\left(\pi^{\prime \prime} G\right)(h)=G(h), h \neq f^{s}, g^{s} \text { for any } s \subseteq\left\{\alpha_{1}, \ldots, \alpha_{n}\right\} .
\end{gathered}
$$

So $\left[\left(\pi^{\prime \prime} G\right)\left(f^{s}\right)\right]=\left[G\left(g^{s}\right)\right],\left[\left(\pi^{\prime \prime} G\right)\left(g^{s}\right)\right]=\left[G\left(f^{s}\right)\right]$, and $\left[\left(\pi^{\prime \prime} G\right)(h)\right]=[G(h)]$ for $h \neq f^{s}, g^{\delta}$.

Thus the change from $G$ to $\pi^{\prime \prime} G$ leaves $X$, and thence $Y$ and $Z$, unchanged, leaves $G\left(f_{1}\right), \ldots, G\left(f_{m}\right)$ unchanged and carries $[G(f)]$ to $[G(g)]$. Take $\alpha \in\left\{\alpha_{1}, \ldots, \alpha_{n}\right\} ;$ for $s \subseteq\left\{\alpha_{1}, \ldots, \alpha_{n}\right\}$ set $s^{\prime}=s \Delta\{\alpha\}$. Then $\left\{\left[G\left(f^{S}\right)\right],\left[G\left(f^{s^{\prime}}\right)\right]\right\} \in y_{\alpha}$ and $\left\{\left[G\left(g^{s}\right)\right],\left[G\left(g^{s^{\prime}}\right)\right]\right\} \in Y_{\alpha}$. The change from $G$ to $\pi^{\prime \prime} G$ carries each of these pairs to the other, so $y_{\alpha}$ is carried into itself.

In conclusion $M[G] \vDash \psi\left(\pi^{\prime \prime} G, y\right) \leftrightarrow N \models \varphi\left(z, y_{\alpha_{1}}, \ldots, y_{\alpha_{n}}, G\left(f_{1}\right), \ldots, G\left(f_{m}\right), x,[G(g)]\right)$. Since $M[G] \vDash \psi\left(\pi^{\prime \prime} G, y\right)$, the proof is complete.

THEOREM 3.5. $N \vDash X$ is QM.

Proof. We work in $N$. Suppose that

$$
N \vDash A \text { is an infinite subset of } X \text {. }
$$

By the remarks after Theorem 3.3 we may assume that $A$ is defined in terms of $2, y_{\alpha_{1}}, \ldots, y_{\alpha_{n}}, G\left(f_{1}\right), \ldots, G\left(f_{m}\right)$ say, and $x \in M$. Take $a \in A-\left\{\left[G\left(f_{i}^{s}\right)\right]: 1 \leq i \leq m\right.$ and $\left.s \subseteq\left\{\alpha_{1}, \ldots, \alpha_{n}\right\}\right\}$. Now $a=[G(f)]$ for some $f$, so the sentence ' $a \in A^{\prime}$ ' may be written in the form 


$$
N \vDash \varphi\left(z, Y_{\alpha_{1}}, \ldots, Y_{\alpha_{n}}, G\left(f_{1}\right), \ldots, G\left(f_{m}\right), x,[G(f)]\right) .
$$

Application of Lemma 3.4 shows that

$$
N \vdash A \supseteq X-\left\{\left[G\left(f_{i}^{s}\right)\right]: 1 \leq i \leq m \text { and } s \subseteq\left\{\alpha_{1}, \ldots, \alpha_{n}\right\}\right\},
$$

so $N \vdash A$ is cofinite.

In conclusion we have shown that for $k$ an arbitrary aleph it is possible to have a QM set $X$ such that $k<|S(S(S(X)))|$. This result is one of a series: Hickman [1] and the author ( $\mathrm{PhD}$ thesis, University of Bristol, 1971) have independently shown that it is possible to have a DF set $X$ such that $K<|S(X)|$ (again for $K$ an arbitrary aleph); indeed Hickman obtains $k<\left|X^{[2]}\right|$. Also the author (unpublished) has shown that it is possible to have a set $X$ such that $X * \omega$ (whence by Lemma 2.2, $S(X)$ is DF) but such that $k<|S(S(X))|$. It should be emphasised that none of these results have anything to do with the possibility that $2^{\omega}$ can be large; in all the models concerned if $k, \lambda$ are alephs then

$$
\kappa \leq 2^{\lambda} \rightarrow \kappa \leq \lambda^{+} \text {. }
$$

\section{References}

[1] J.L. Hickman, "On the set of finite subsets of a set", submitted.

[2] Herman Rubin and Jean E. Rubin, Equivalents of the axiom of choice (North-Holland, Amsterdam, 1963).

[3] J.R. Shoenfield, "Unramified forcing", Axiomatic set theory, 357-381

(Proc. Sympos. Pure Math., Vol. 13, Part 1, Univ. California, Los Angeles, Calif., 1967; Amer. Math. Soc., Providence, Rhode Island, 1971).

[4] Alfred Tarski, "Sur les ensembles finis", Fund. Math. 6 (1924), 45-95.

Department of Pure Mathematics, University of Sydney, Sydney, New South Wales. 